\title{
Influencia del vetiver y eucalipto en la estabilidad de taludes
}

\section{Influence of Vetiver and Eucalyptus on slopes stability}

\author{
Luis David Chaparro-Sarmiento ${ }^{\text {1a }}$, Wilmer Josué Castañeda-Quijano ${ }^{\text {1b }}$, \\ Óscar F. Sánchez-Ortiz ${ }^{1 c}$ \\ ${ }^{1}$ Grupo de investigación Geomática, Gestión y Optimización de Sistemas, Escuela de Ingeniería Civil, Universidad

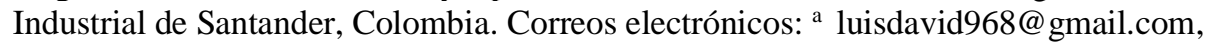 \\ b wijo_9@ hotmail.com, ${ }^{\mathrm{c}}$ oscar.sanchez2@ correo.uis.edu.co. Orcid: ${ }^{\mathrm{a}}$ 0000-0002-0424-842X, \\ ${ }^{\mathrm{b}}$ 0000-0002-5516-8412, ${ }^{\mathrm{c}} 0000-0002-9185-468 \mathrm{X}$.
}

Recibido: 29 diciembre, 2020. Aceptado: 17 junio, 2021. Versión final: 2 junio, 2021.

\section{Resumen}

El eucalipto y el vetiver son plantas comunes en Colombia, las cuales pueden ser tomadas en cuenta por su efecto estabilizador por los ingenieros locales. Se realizó el análisis del efecto en la estabilidad que produce el eucalipto y el vetiver sobre diferentes tipos de suelos, variando la geometría, el ángulo de inclinación y teniendo en cuenta el efecto del nivel freático. El método de análisis trabajado es el equilibrio límite bajo el criterio de Mohr-Coulomb. Los efectos de las raíces mostrados en cohesión añadida varían dependiendo de la profundidad en el suelo y del tipo de planta; estos junto con el peso del eucalipto son los parámetros que se tuvieron en cuenta en el uso de la vegetación como agente estabilizador de taludes. El efecto del agua sobre el talud se puede ver en los resultados, y tiene un impacto significativo en el factor de seguridad del talud, sobre todo cuando los ángulos de inclinación son bajos y dependiendo del tipo de vegetación presente. Finalmente, se demostró la efectividad del eucalipto en la estabilidad de taludes, cuyo único factor negativo es su peso en altas pendientes, mientras que el aporte ofrecido por el vetiver joven a la estabilidad del talud no es muy significativo.

Palabras clave: estabilidad de taludes; eucalipto; vetiver; factor de seguridad; vegetación; raíces; equilibrio límite.

\begin{abstract}
At the local ambit, eucalyptus and vetiver are common plants in Colombia, which can consider by their stabilizing effect by local engineers. It was carried out an analysis of the effect on stability produced by eucalyptus and vetiver on different kinds of soils, varying the geometry, the inclination angle, and considering the water table effect. The method of analysis used is the limit equilibrium under the Mohr-Coulomb criteria. The root effect shown in cohesion added varies depending on soil depth, and plant type, as well as the weight of eucalyptus, are the parameters that are considered in the use of vegetation as a slope stabilizing agent. The effect of water on the slope can be seen in the results, being significant in the slope safety factor, especially when the inclinations angles are low and affected by the type of vegetation present. Finally, the effectiveness of eucalyptus in slope stability was demonstrated, the only negative factor being its weight on high slopes, while the contribution offered by the young vetiver to the stability of the slope is not very significant.
\end{abstract}

Keywords: slope stability; Eucalyptus; Vetiver; security factor; vegetation, roots; limit equilibrium.

ISSN impreso: 1657 - 4583. ISSN en línea: 2145 - 8456, CC BY-ND 4.0 @) (1) 을

Como citar: L. D. Chaparro-Sarmiento, W. J. Castañeda-Quijano, O. F. Sánchez-Ortiz, "Influencia del vetiver y eucalipto en la estabilidad de taludes" Rev. UIS Ing., vol. 20, no. 4, pp. 171-188, 2021, doi: 10.18273/revuin.v20n42021014. 


\section{Introducción}

La vegetación que crece a lo largo de un talud ha adquirido intereses investigativos por sus efectos o influencias sobre el mismo; además, se contemplan otros factores como las precipitaciones, presencia de aguas subterráneas y la deforestación en regiones montañosas y laderas de ríos [1], [2], que están causando preocupación en muchas partes del mundo debido a que aumentan la probabilidad de deslizamientos de tierra y dificultan aún más controlar la estabilidad del talud [3].

Varios estudios han relacionado la disminución de la cohesión aparente del suelo con la pérdida de raíces [4], [5], lo que significa una alteración de la estabilidad de una ladera cuando se presentan grandes fenómenos de deforestación en la zona, ya que esta queda vulnerable a fallas por erosión masiva de materiales y posibilita la generación de una catástrofe. Un buen ejemplo fue lo sucedido en el departamento de Putumayo. Esta catástrofe tuvo lugar en Mocoa donde fuertes lluvias provocaron el desbordamiento de los ríos Mocoa, Sangoyaco y Mulato, los cuales, al ser fuentes fluviales torrenciales, arrastraron a su paso sedimentos, basuras y piedras descomunales que provocaron una enorme avalancha que dejó 254 muertos e innumerables daños de bienes. Esta catástrofe se originó en la deforestación masiva de vegetación en esta zona, ya que al no existir esa masa boscosa que sirve para contener las aguas y darle firmeza a los suelos, se facilita las remociones en masa [6].

La investigación sobre la influencia de las raíces de las plantas en las pendientes se ha venido desarrollando con auge en el último tiempo [7], [8], [9] en búsqueda de una relación amigable con el medio ambiente. Diferentes plantas (hierba, plantas regionales, árboles regionales, etc.) pueden ofrecer una solución eficiente ante las problemáticas que pueden afectar una ladera, ya que refuerzan y estabilizan el talud [10].

En Colombia se han hecho pocos estudios al respecto, ya que no se ha visto de una manera técnicamente viable, pero en el país hay diferentes profesionales capacitados como ingenieros forestales, hidrólogos y geotecnistas que pueden investigar cómo las plantas pueden mejorar la estabilidad de taludes [11]. A pesar del hecho de que numerosas investigaciones se han llevado a cabo para comprender las interacciones vegetación-pendiente [9], [12], desafortunadamente en la estabilidad de laderas se da poca consideración a la vegetación para aplicaciones de ingeniería geotécnica; por desconocimiento generalizado, resulta muchas veces discutido, al punto que pueden encontrarse tanto defensores como detractores [13].
La vegetación que crece en una pendiente puede ser de diferentes tipos: pasto, arbustos, árboles o una combinación de dos o tres tipos de vegetación [14]. Entre los parámetros mecánicos de la vegetación, la estabilización mecánica debido a la presencia de raíces y el recargo por el peso de los árboles [12] son los que se consideran la mayor parte del tiempo.

Hay varios tipos de vegetación en Colombia que pueden aplicarse a una pendiente como lo son Eucalyptus o eucalipto [15], como se llama ordinariamente: una especie de crecimiento rápido, con vida relativamente corta. Se dice que su sistema radicular es superficial, pero hay raíces pivotantes a profundidades mayores de $2 \mathrm{~m}$. El mayor desarrollo radicular está entre los $15-45 \mathrm{~cm}$ de suelo, y cerca del $96 \%$ de las raíces se concentra en los primeros $60 \mathrm{~cm}$. Se reportan raíces principales hasta de 8 $\mathrm{m}$, con diámetros promedios de $20 \mathrm{~cm}$, y pocas raíces secundarias [16].

Otra planta caso de estudio es el vetiver, científicamente llamado Chrysopogon zizanioides [17], [18]. Su sistema masivo de raíces en general crece verticalmente y no compite con los cultivos adyacentes. En condiciones buenas, el sistema radial puede crecer hasta una profundidad de $4 \mathrm{~m}$, y bajo la superficie del suelo se forma una barrera. Las raíces son muy fuertes y tienen la capacidad de amarrar la sarta junta [19].

Estas dos plantas son el objetivo de este estudio, ya que son muy comunes en el ámbito, son de fácil adquisición y de gran resistencia a climas drásticos como los de Colombia [20]. El objetivo del presente estudio es cuantificar el efecto de la geometría de la pendiente, así como los parámetros mecánicos de dos tipos de vegetación modelados sobre la estabilidad de una pendiente con y sin los efectos generados bajo las condiciones de nivel freático. Se utilizan cálculos de equilibrio límite en el software Slide 7.0 bajo el método de Spencer (se asume que la inclinación de las fuerzas laterales son las mismas para cada tajada. Rigurosamente satisface el equilibrio estático asumiendo que la fuerza resultante entre tajadas tiene una inclinación constante pero desconocida). Además, se realiza un análisis de sensibilidad simple para evaluar el factor de seguridad en una pendiente para las variaciones de la vegetación y presencia de un nivel freático localizado a una determinada altura.

\section{Materiales y metodología}

Se tuvieron en cuenta dos tipos de plantas, la presencia de agua subterránea y cuatro variedades de suelos como factores que afectan la estabilidad de los diferentes tipos 
de taludes. El análisis se realizó mediante el método de equilibrio límite y mediante el criterio de falla, según la teoría de Mohr-Coulomb usando el programa Slide 7.0 de Rocscience bajo las condiciones de cálculo de Spencer.

\subsection{Análisis del talud por el método de equilibrio límite}

En el estudio se modelaron los suelos (véase tabla 1) tomados de [10], [21]; a cada suelo se le añadió paulatiamente la cohesión de las plantas con respecto a la profundidad, tomadas de [22], [23]. La geometría en 2D se obtuvo de [10]. Spencer fue el método que se consideró para este análisis con una cantidad de 50 dovelas verticales, una tolerancia de 0,005 y un total de 75 interaciones.

El N. F. (nivel freático) se modeló mediante el método steady state FEA, cabeza total, y se trabajó con un peso específico del agua de $9,81 \mathrm{kN} / \mathrm{m}^{3}$; esto se realizó con la mitad de los análisis para tener en cuenta el mismo talud con y sin presión de poros. Se creó la malla con una cantidad de 20 intevalos en los ejes principales (X, Y).

Para el eucalipto [22] se consideró su propio peso, por tanto, se le añadió una carga distribuida a los modelos, donde se usó dicha planta, aunque también se analizó el efecto de la carga del eucalipto sin considerar la cohesión añadida al talud.

\subsection{Suelos}

En el análisis se necesitaron los parámetros del ángulo de rozamiento $\left(\varphi^{\prime}\right)$, el peso específico y la cohesión del suelo. Se tomaron los suelos de arena, arcilla y arena limosa de [10], y el suelo de arcilla limosa de [21].

Utilizando el criterio de Mohr Coulomb se analizó la resistencia al corte de cada suelo:

$$
\tau=c+\sigma * \tan (\varphi)
$$

Donde $\tau$ es la resistencia al corte del suelo, $c$ es la cohesión del suelo, $\sigma$ es el esfuerzo normal del suelo y $\varphi$ es el ángulo de fricción interna.

\subsection{Diseño del talud}

La geometría [10] está basada en dos parámetros que definen las caracterísiticas del talud: $\mathrm{H}$, medido en metros que rije la altura, el largo de la geometría y la profundidad del nivel freático; y $\alpha$ como el ángulo de inclinación (véase figura 1).
Tabla 1. Propiedades mecánicas de los diferentes tipos de suelos como objetivo de análisis

\begin{tabular}{|c|c|c|c|}
\hline Suelo & $\begin{array}{c}\text { Densidad } \\
\text { drenada (kPa) }\end{array}$ & $\begin{array}{c}\text { Cohesión } \\
(\mathbf{k P a})\end{array}$ & $\begin{array}{c}\text { Ángulo de } \\
\text { fricción } \boldsymbol{\varphi} \\
\left({ }^{\circ}\right)\end{array}$ \\
\hline $\begin{array}{c}\text { Arcilla } \\
\text { limosa }\end{array}$ & 14,4 & 6,75 & 33,6 \\
\hline $\begin{array}{c}\text { Arena } \\
\text { limosa }\end{array}$ & 17 & 2 & 25 \\
\hline Arena & 17 & 0,001 & 30 \\
\hline Arcilla & 16 & 2 & 20 \\
\hline
\end{tabular}

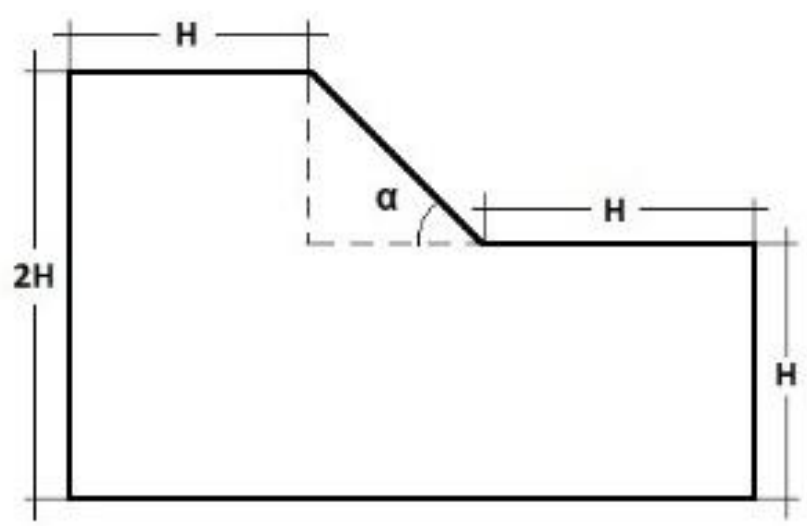

Figura 1. Dimensionamiento del talud donde $\mathrm{H}$ tiene valores de $5,10,15$ y $20 \mathrm{~m}$ y $\alpha$ es el ángulo del talud $\left(11^{\circ}, 14^{\circ}, 19^{\circ}, 22^{\circ}, 27^{\circ}, 34^{\circ}, 45^{\circ}\right)$.

\subsection{Vegetación}

Dos plantas existentes en Colombia como son el árbol eucalipto y el pasto vetiver se consideraron como agente estabilizador del talud que proporcionan cohesión por medio de sus raíces. El árbol eucalipto genera una cohesión adicional de $10 \mathrm{kPa}$ hasta $2 \mathrm{~m}$ de profundidad, de $5 \mathrm{kPa}$ entre 2 y $4 \mathrm{~m}$ de profundidad y de $2 \mathrm{kPa}$ entre 4 y $6 \mathrm{~m}$ de profundidad [24] (véase figura 2); el peso del eucalipto se tuvo en cuenta y se tomó el valor de $2,5 \mathrm{kPa}$ [25].

La cohesión añadida que proporciona el pasto vetiver, muy usado en el mundo como planta estabilizadora de taludes, fue tomada de [25], quien indica que hasta una profundidad de $30 \mathrm{~cm}$ se genera un $\mathrm{Cr}$ de $2,5 \mathrm{kPa}$ (véase figura 3). 


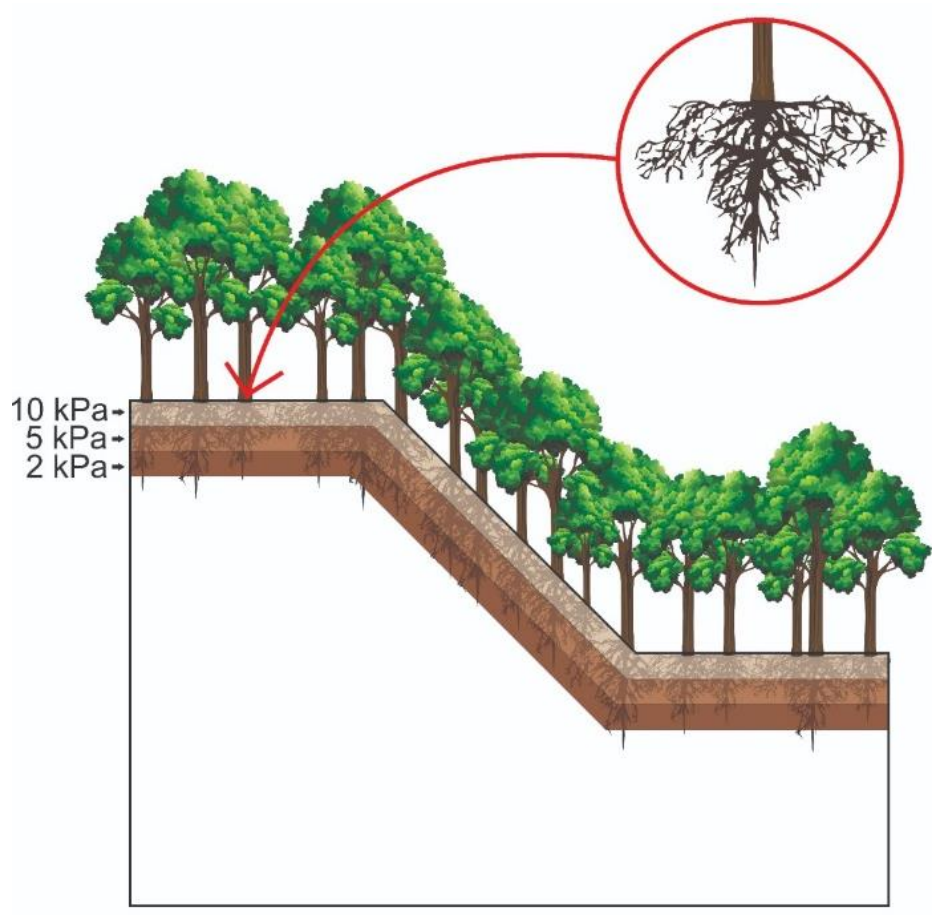

Figura 2. Reforzamiento de las raíces del eucalipto basado en niveles, cada capa cuenta con $2 \mathrm{~m}$ de profundidad desde la superficie hasta un total de $6 \mathrm{~m}$.

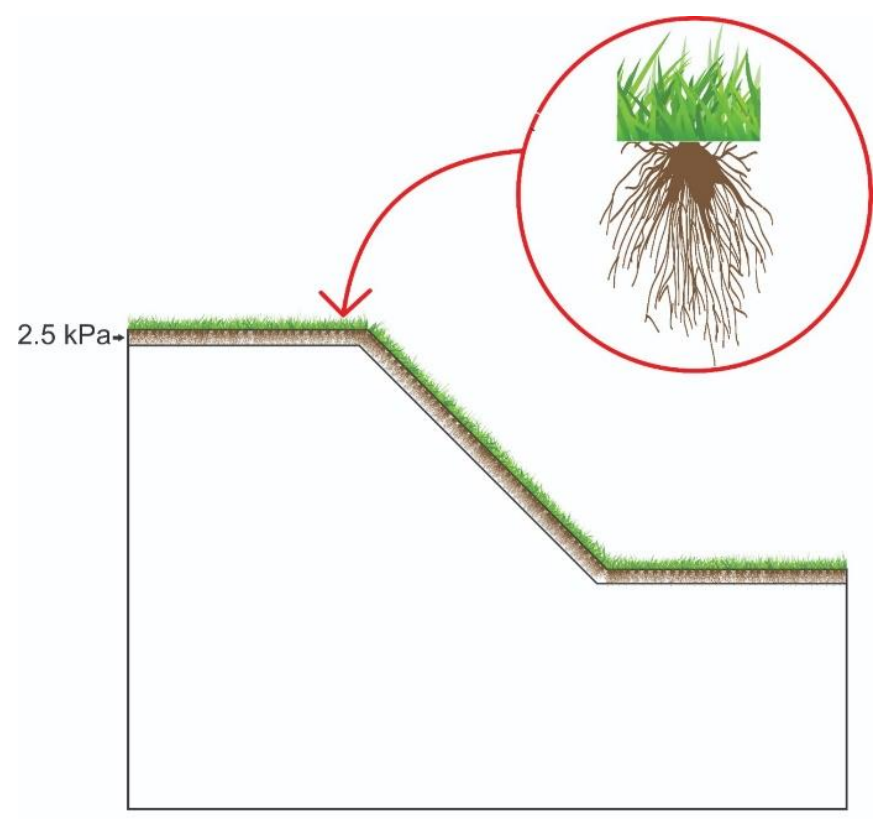

Figura 3. Reforzamiento de las raíces vetiver cuyo aporte es generado a una distancia de $2 \mathrm{~m}$ con respecto de la superficie del talud. 


\subsection{Nivel freático}

Se estimó un nivel freático cuya altura dependía de la geometría, con $80 \%$ de $\mathrm{H}$ para el borde más bajo, el cual está cerca del pie del talud, y un $70 \%$ de $2 \mathrm{H}$ para el borde más alto, el cual está en la corona del talud (véase figura $4)$.

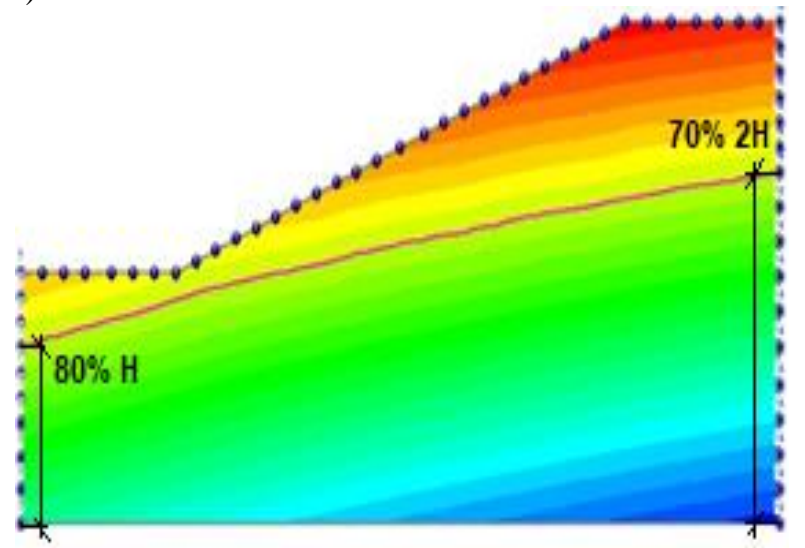

Figura 4. Nivel freático del talud mediante el método steady state FEA, teniendo en cuenta la carga hidráulica total.

\section{Resultados}

A continuación, se muestra el comportamiento de los diferentes tipos de suelos (arcilla, arcilla limosa, arena limosa) a los que se les aplicó el refuerzo de las plantas con presencia de nivel freático y sin este.

\subsection{Comportamiento de los diferentes tipos de suelos}

\subsubsection{Arcilla limosa}

Se determinó que el comportamiento de las arcillas limosas fue el menos eficiente en la estabilidad del talud al reforzarse con las diferentes plantas, ya que, como se observa en la figura 5, fue donde presentó el menor incremento del factor de seguridad (F. S.) comparado con los otros tipos de suelos (arena, arena limosa, arcilla). Al implementar la planta vetiver se presentó un comportamiento casi nulo o insignificante en este suelo como se puede observar en la tabla 2 (condiciones de presencia de agua en el talud) y en la tabla 3 (en condiciones secas).

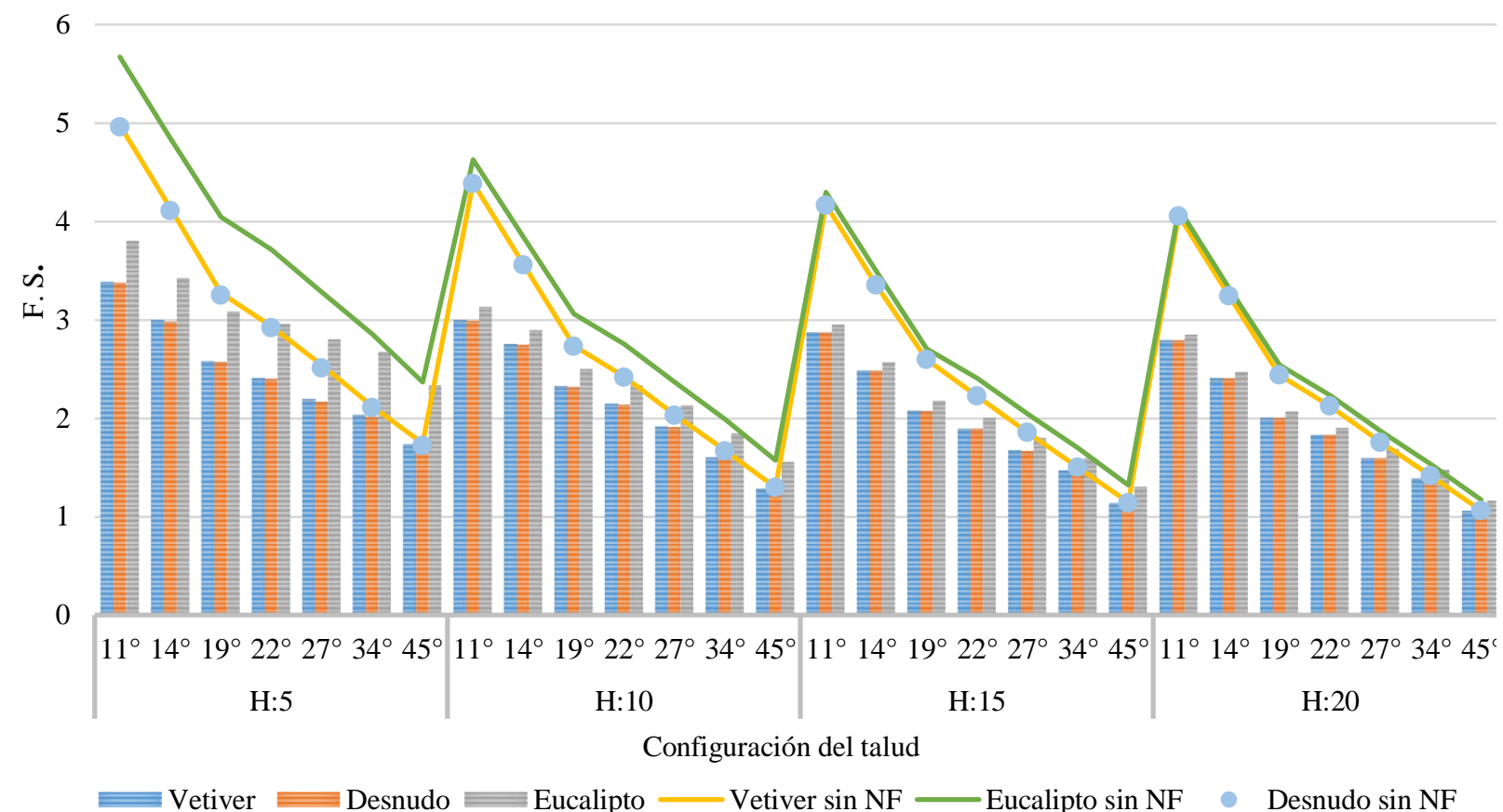

Figura 5. Comportamiento de los factores de seguridad de la arcilla limosa en diferentes ángulos de inclinación del talud y para diferentes geometrías (H: $5,10,15,20)$, con influencia de diferentes plantas (vetiver, eucalipto) y sin estas (desnudo). 
Con el uso del eucalipto como refuerzo se observó una mejoría en las arcillas limosas con un incremento del factor de seguridad en un promedio del 12,27\% comparado con un talud de suelo arcillo limoso sin refuerzo alguno y en presencia del nivel freático en promedio de $11,25 \%$ (véanse tabla 2 y 3 ). Al compararse con los otros tipos de suelo, otorga el menor incremento del factor de seguridad sobre el talud, a pesar de que este tipo de suelo fue el que presentó el mayor factor de seguridad comparado con los otros suelos desnudos y con diferentes configuraciones del talud, tanto en presencia de agua y sin esta, por lo tanto, como el factor de seguridad es alto, el incremento relativo del F. S. es menor.

Tabla 2. Comportamiento medio de la arcilla limosa en presencia de agua

\begin{tabular}{|c|c|c|c|}
\hline \multicolumn{4}{|c|}{ \% Arcilla limosa con N. F. } \\
\hline Planta & Desnudo & Vetiver & Eucalipto \\
\hline$\Delta$ F. S. & - & $0,330 \%$ & $11,255 \%$ \\
\hline F. S. & 2.100 & 2.109 & 2.339 \\
\hline
\end{tabular}

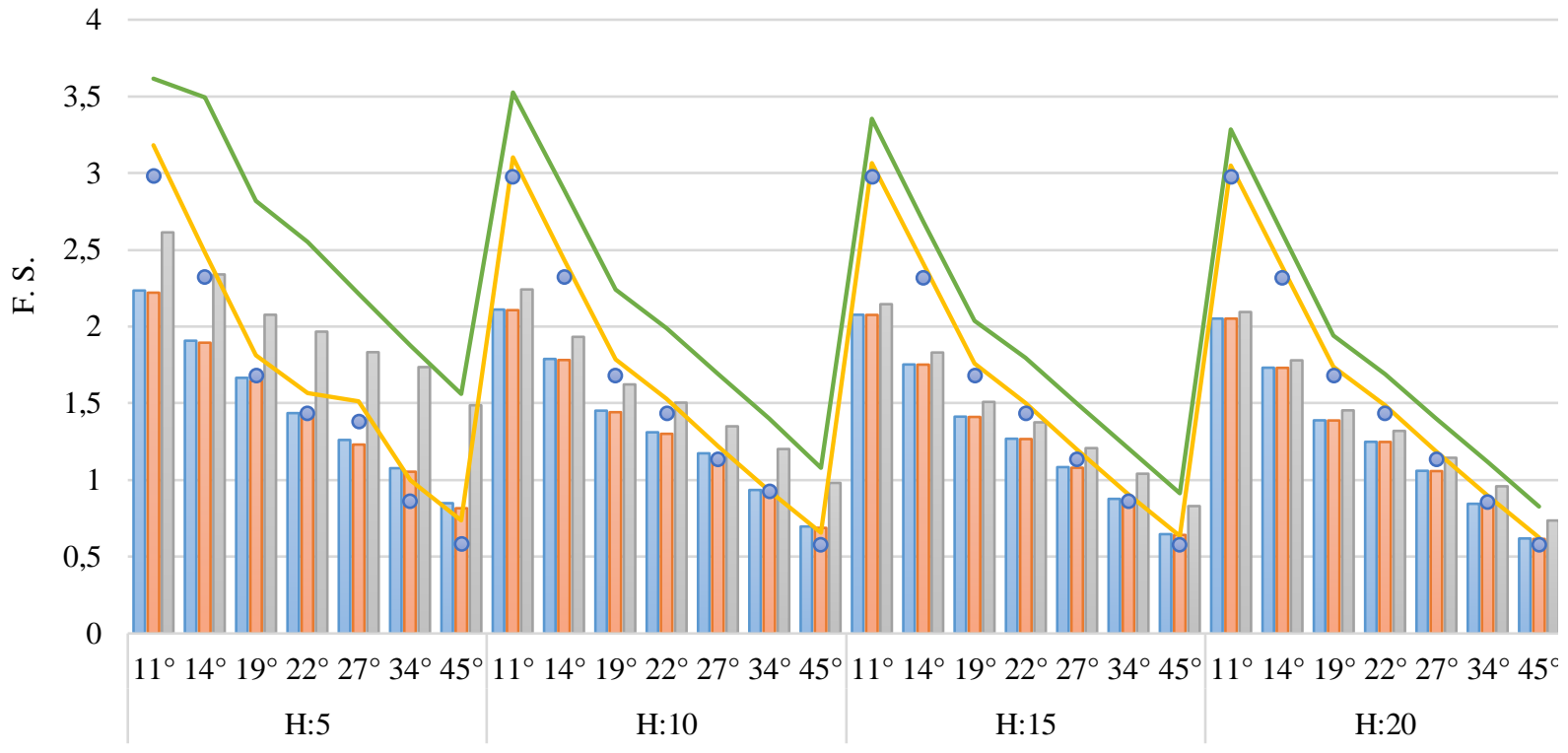

Configuración del talud

$\square$ Vetiver $\square$ Desnudo $\square$ Eucalipto $\quad$ Vetiver sin NF $\quad$ Eucalipto sin NF $\circ$ Desnudo sin NF

Figura 6. Comportamiento de los factores de seguridad de la arena limosa en diferentes ángulos de inclinación del talud en diferentes geometrías (H: 5, 10, 15, 20), con influencia de diferentes plantas (vetiver, eucalipto) y sin estas (desnudo).

\section{\% Arcilla limosa sin N. F.}

\begin{tabular}{|c|c|c|c|}
\hline Planta & Desnudo & Vetiver & Eucalipto \\
\hline F. S. & 2.610 & 2.610 & 2.919 \\
\hline F. S. & - & $0,393 \%$ & $12,278 \%$ \\
\hline
\end{tabular}

\subsubsection{Arena limosa}

La arena limosa ante el refuerzo de la planta vetiver tuvo un comportamiento similar al de la arcilla limosa, ya que el incremento del factor de seguridad fue muy pequeño,

pero este tuvo un leve incremento comparado al suelo arcillo limoso, aunque no le aportó significativamente una mejora al comportamiento de seguridad del talud.

Como se ilustra en la figura 6, el comportamiento del vetiver no se ve fuertemente afectado por las diferentes geometrías y pendientes del talud; se observa que su mejor factor de seguridad se encuentra en el talud de geometría más pequeña y pendiente de menor inclinación $\left(11^{\circ}\right)$. 
En cambio, al reforzar la arena limosa con la planta eucalipto se obtuvo un mejor aporte del F. S., con un incremento del factor de seguridad del $22,85 \%$ y en presencia de nivel freático de $17,25 \%$, dando así un incremento para la confiabilidad del talud como se observa en las tablas 4 y 5 , que hace que el eucalipto se comporte de una manera más confiable con las arenas limosas en geometrías pequeñas como $\mathrm{H}=5 \mathrm{~m}$ y $\mathrm{H}=10$ $\mathrm{m}$.

Con el uso del vetiver como refuerzo en el talud se obtuvo un comportamiento aceptable cuando se modeló con la menor geometría y pendiente; se obtuvieron factores de seguridad de 2,31 con niveles freáticos y un factor de seguridad de 2,97 sin presencia de agua.
Tabla 4. Comportamiento medio de la arena limosa en presencia de agua

\% Arenas limosa con N. F.

\begin{tabular}{|c|c|c|c|}
\hline Planta & Desnudo & Vetiver & Eucalipto \\
\hline$\Delta$ F. S. & - & $0,741 \%$ & $17,246 \%$ \\
\hline F. S. & 1.350 & 1.360 & 1.583 \\
\hline
\end{tabular}

Tabla 5. Comportamiento medio de la arena limosa en condiciones secas

\begin{tabular}{|c|c|c|c|}
\hline \multicolumn{4}{|c|}{ \% Arenas limosa sin N. F. } \\
\hline Planta & Desnudo & Vetiver & Eucalipto \\
\hline$\Delta$ F. S. & - & $0,803 \%$ & $22,845 \%$ \\
\hline F. S. & 1.548 & 1.560 & 1.901 \\
\hline
\end{tabular}

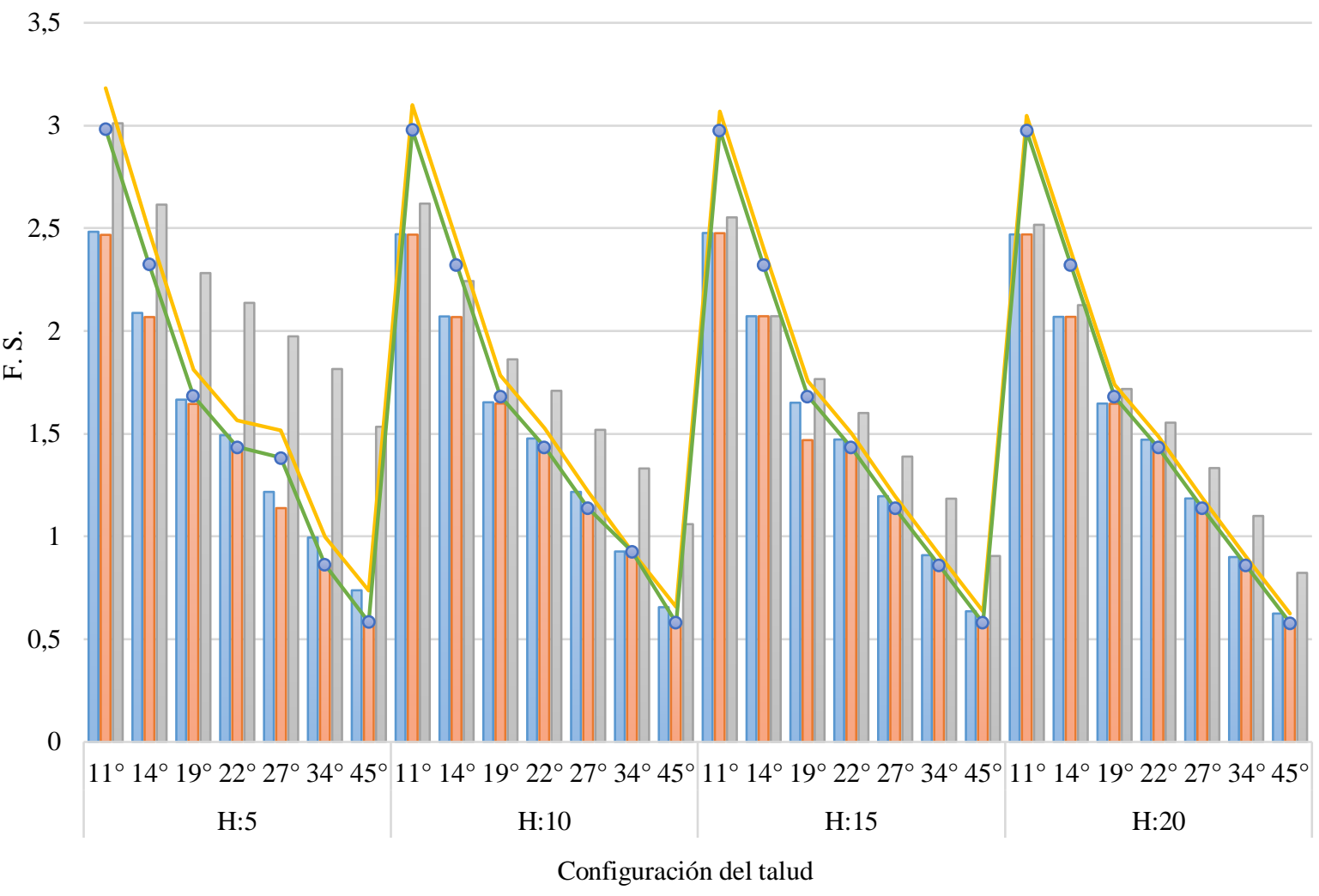

$\square$ Vetiver $\square$ Desnudo $\square$ Eucalipto $\quad$ Vetiver sin NF $\quad$ Eucalipto sin NF $\circ$ Desnudo sin NF

Figura 7. Comportamiento de los factores de seguridad de la arena en diferentes ángulos de inclinación del talud en diferentes geometrías (H: 5, 10, 15, 20), con influencia de diferentes plantas (vetiver, eucalipto) y sin estas (desnudo). 


\subsubsection{Arena}

Se determinó que la arena fue el suelo donde el refuerzo del vetiver fue más influyente, comparado con los otros tipos de suelo (véase figura 7), debido a que la falla en arenas es superficial, lo que genera que el vetiver sí influya a pesar de que sea de raíz somera, comparado con el suelo sin ningún refuerzo aplicado (véanse tablas 6 y 7). Su comportamiento no se vio fuertemente influenciado por las diferentes geometrías, pero sí influyó su ángulo de fricción, ya que al no haber cohesión (véase tabla 1), el factor de seguridad se ve afectado directamente por el ángulo de fricción y por el esfuerzo cortante que afecta el factor de seguridad del talud.

Igualmente, el refuerzo del eucalipto en arenas presentó su mayor incremento en el factor de seguridad, como se puede ver en las tablas 6 y 7 ; se obtuvo un incremento del factor de seguridad en un $33,87 \%$ y $23,75 \%$ en presencia de aguas subterráneas.
A diferencia del vetiver, la geometría sí influyó en la arena con refuerzo de eucalipto, ya que el talud reforzado presentó un F. S. más alto, por ende, más seguro, sobre todo cuando el talud tuvo la menor geometría $(\mathrm{H}=5)$, y las geometrías de mayor tamaño tienden a tener un comportamiento similar entre ellas (desnudo, eucalipto, vetiver) como en el caso $\mathrm{H}=15 \mathrm{~m}, 20 \mathrm{~m}$. Se observó que su comportamiento óptimo en cuanto a factor de seguridad es la geometría de $\mathrm{H}=5 \mathrm{~m}$, con una pendiente de $11^{\circ}$. Sin presencia de agua obtuvo un factor de seguridad de 3,63; este es el mayor factor de seguridad obtenido.

Tabla 6. Comportamiento medio de la arena húmeda

\begin{tabular}{|c|c|c|c|}
\hline \multicolumn{4}{|c|}{ \% Arenas con N. F. } \\
\hline Planta & Desnudo & Vetiver & Eucalipto \\
\hline$\Delta$ F. S. & - & $3,057 \%$ & $23,754 \%$ \\
\hline F. S. & 1.453 & 1.498 & 1.798 \\
\hline
\end{tabular}

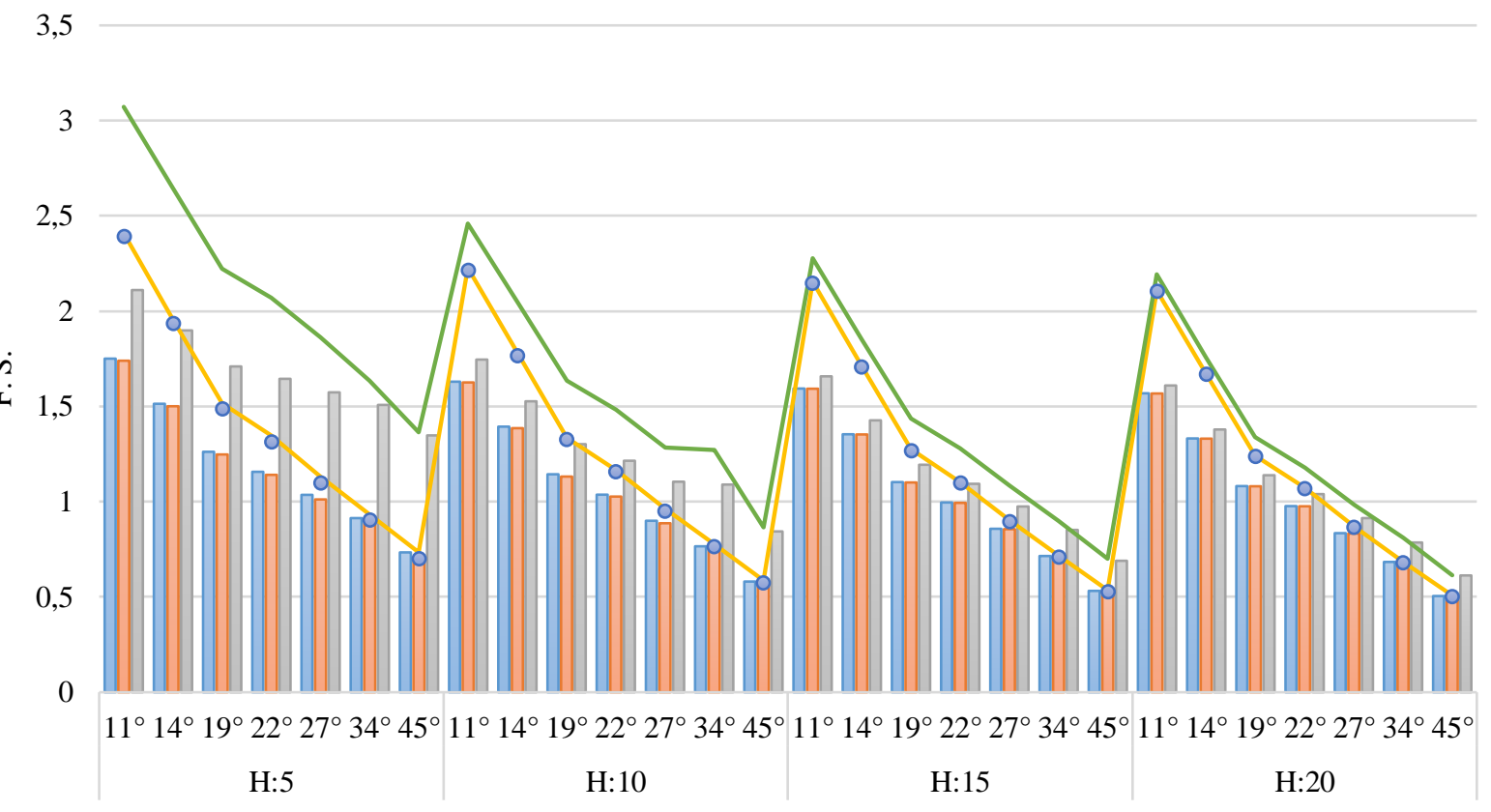

Configuración del talud

$\square$ Vetiver $\square$ Desnudo $\square$ Eucalipto $\quad$ Vetiver sin NF $\quad$ Eucalipto sin NF $\circ \quad$ Desnudo sin NF

Figura 8. Comportamiento de los factores de seguridad de la arcilla en diferentes ángulos de inclinación del talud en diferentes geometrías (H: 5, 10, 15, 20), con influencia de diferentes plantas (vetiver, eucalipto) y sin estas (desnudo) bajo condiciones de nivel freático. 
Tabla 7. Comportamiento medio de la arena seca

\begin{tabular}{|c|c|c|c|}
\hline \multicolumn{4}{|c|}{$\%$ Arenas sin N. F. } \\
\hline Planta & Desnudo & Vetiver & Eucalipto \\
\hline$\Delta$ F. S. & - & $5,602 \%$ & $33,870 \%$ \\
\hline F. S. & 1.582 & 1.671 & 2.118 \\
\hline
\end{tabular}

\subsubsection{Arena}

Las arcillas comparadas con los otros diferentes tipos de suelos son las que presentan el menor factor de seguridad (véase figura 8). Al reforzar las arcillas con eucalipto se presentó un incremento medio del $26,20 \%$ (véase tabla 9), y en presencia de agua, de un 20,54 \% (véase tabla 8), esto da un incremento sustancial al talud. Por el contrario, el vetiver obtuvo un bajo factor de seguridad.

La presencia de niveles freáticos en el talud fue un factor importante en las arcillas, ya que hacían disminuir drásticamente el factor de seguridad comparado al F. S. sin presencia de niveles freáticos. Como en el eucalipto se obtuvieron F. S. de 3,15, y cuando interactuó con niveles freáticos se disminuyó el F. S. a 2,21, con una disminución del $33 \%$ de su F. S. inicial, en las 2 plantas de estudio se presentó un comportamiento muy similar de disminución del F. S., haciendo de este un factor crítico en las arcillas.

Tabla 8. Comportamiento medio de la arcilla en presencia de agua

\section{\% Arcillas con N. F.}

\begin{tabular}{|c|c|c|c|}
\hline Planta & Desnudo & Vetiver & Eucalipto \\
\hline$\Delta$ F. S. & - & $0,770 \%$ & $20,548 \%$ \\
\hline F. S. & 1.062 & 1.070 & 1.280 \\
\hline
\end{tabular}

Tabla 9. Comportamiento medio de la arcilla en condiciones secas

\% Arcillas sin N. F.

\begin{tabular}{|c|c|c|c|}
\hline Planta & Desnudo & Vetiver & Eucalipto \\
\hline$\Delta$ F. S. & - & $1,250 \%$ & $26,204 \%$ \\
\hline F. S. & 1.254 & 1.270 & 1.583 \\
\hline
\end{tabular}

\subsection{Análisis comparativo de suelos y su influencia con el nivel freático}

Como se observa en la tabla 10, cuando se aplican los refuerzos de las plantas a los suelos en presencia de niveles freáticos, mejora su F. S.

El vetiver fue el que se vio menos influenciado por el agua, ya que hizo que su factor de seguridad no aumentara considerablemente; en algunos casos como en las arcillas limosas y arenas limosas tuvo un comportamiento promedio igual a un talud sin refuerzo alguno (desnudo).

El refuerzo con plantas de eucalipto presentó un aumento medio del F. S. en presencia de niveles freáticos; comparado con el vetiver, aumentó su factor de seguridad considerablemente tanto en un suelo sin refuerzo alguno como en suelos arenosos reforzados con eucalipto con influencia del agua, lo cual hizo que el F. S. fuera el doble comparado con un talud a la intemperie (desnudo) (véanse tabla 10 y figura 9).

Tabla 10. Influencia del factor de seguridad medio en presencia de nivel freático en diferentes suelos

\begin{tabular}{|c|c|c|c|}
\hline \multicolumn{4}{|c|}{ Influencia del nivel freático en el F. S. [Unidades] } \\
\hline Suelo Planta & Desnudo & Vetiver & Eucalipto \\
\hline $\begin{array}{c}\text { Arcilla } \\
\text { limosa }\end{array}$ & 0,50 & 0,50 & 0,58 \\
\hline Arcilla & 0,19 & 0,20 & 0,30 \\
\hline Arena & 0,13 & 0,17 & 0,32 \\
\hline $\begin{array}{c}\text { Arena } \\
\text { limosa }\end{array}$ & 0,20 & 0,20 & 0,32 \\
\hline
\end{tabular}

\subsection{Efectos de las plantas en los taludes}

Las plantas vetiver y eucalipto usadas como agentes de refuerzo para las diferentes geometrías de taludes generaron un cambio en el factor de seguridad de cada tipo de suelo. En la figura 9 se muestra el incremento del factor de seguridad con respecto a la presencia de nivel freático teniendo en cuenta el efecto de las plantas. La figura 10 representa un promedio del efecto del eucalipto en la estabilidad de los taludes. 


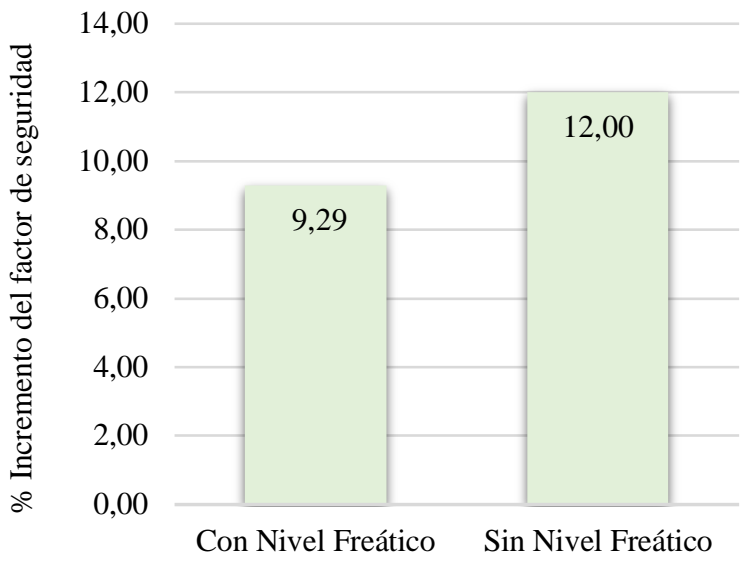

Figura 9. Incremento del factor de seguridad promedio de los taludes analizados debido a la acción de la vegetación (vetiver y eucalipto) con respecto al suelo desnudo; los números son representados en porcentaje.

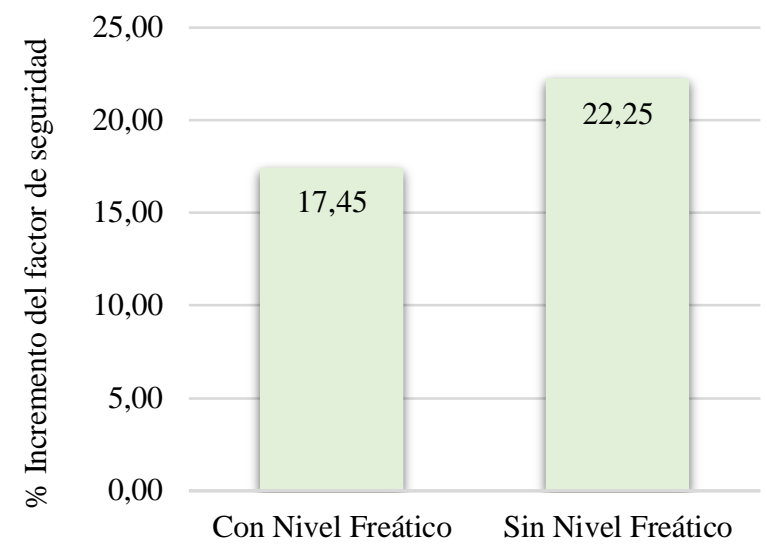

Figura 10. Aumento del factor de seguridad debido a la acción del eucalipto comparado con el suelo desnudo; los números representan porcentajes.

En ausencia del nivel freático, el suelo reforzado con raíces de eucalipto (figura 11) influye en un incremento del $17,45 \%$ y de $22,25 \%$ en promedio del factor de seguridad.

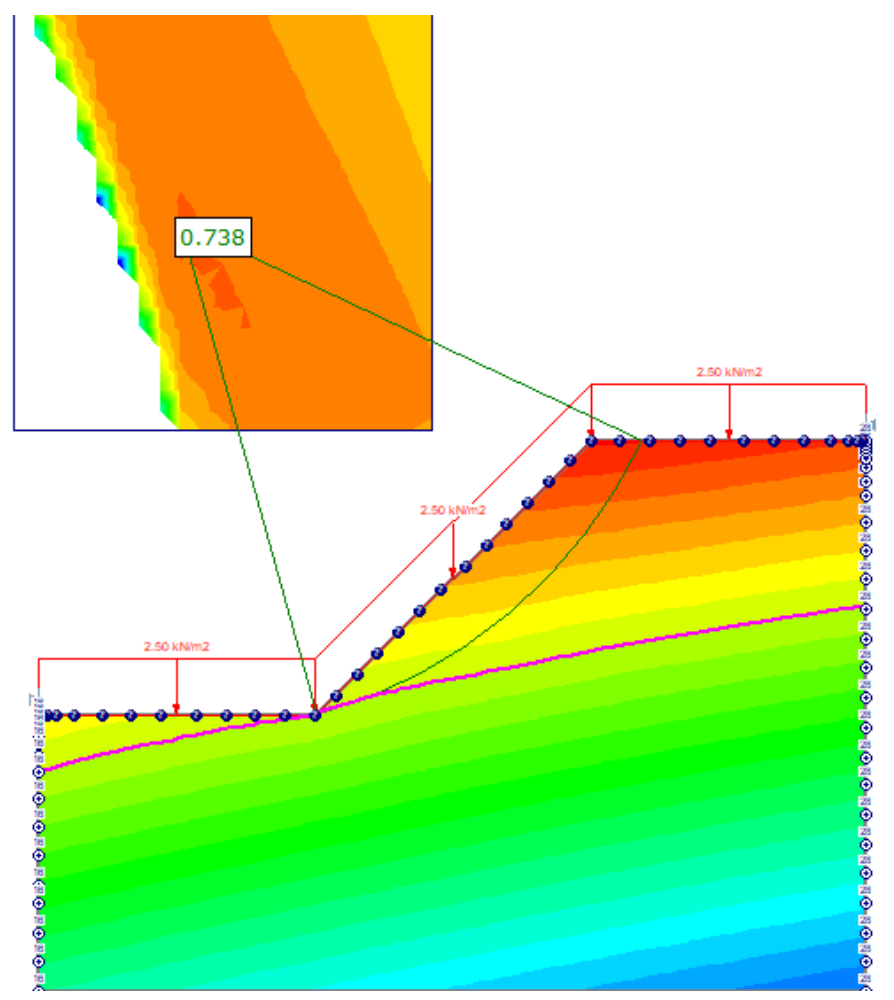

Figura 11. Comportamiento de la falla presente en el talud en presencia de nivel freático reforzado con eucalipto $(2,50 \mathrm{KN} / \mathrm{m} 2)$. 
El vetiver, actuando como agente estabilizador por el aporte de cohesión en promedio al suelo mediante sus raíces, añadió un 1,14\% de factor de seguridad bajo nivel freático y $1,76 \%$ sin este (figura 12).

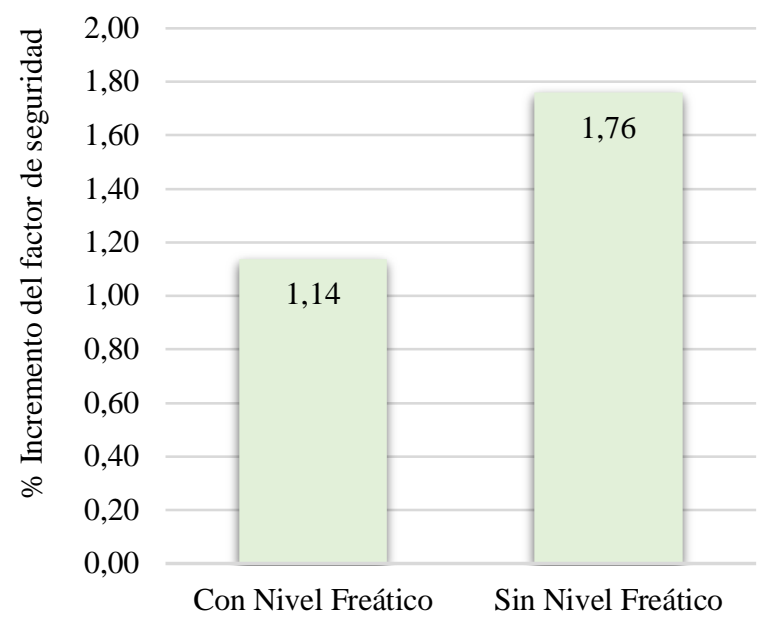

Figura 12. Efectos del vetiver en la estabilidad de taludes comparado con el suelo desnudo; los valores son mostrados en porcentaje.

\subsection{Respuesta del factor de seguridad a la carga impuesta por el eucalipto}

El modelamiento de los diferentes tipos de taludes mediante Slide con las geometrías propuestas en la figura 1 , los suelos descritos en la tabla 1 y usando la carga aplicada por el eucalipto al talud de $2,5 \mathrm{kN} / \mathrm{m}^{2}$ sin tener en cuenta la cohesión añadida por esta planta en los análisis, provoca diferentes resultados en cada tipo de suelo [10]. Se observó el efecto de la carga en los análisis donde se tuvo en cuenta el nivel freático (véase figura 13).

En la arcilla limosa, el ángulo donde la carga dejó de aportar positivamente al talud fue entre los 27 y $34^{\circ}$; para la arena limosa, entre 27 y $34^{\circ}$. En cuanto a la arena el rango de ángulos donde se muestra el paso de ser efectivo a ser un efecto dañino está entre 19 y $22^{\circ}$, y para la arcilla fue entre 27 y $34^{\circ}$.

En la figura 13, el suelo a base de arcilla limosa al aplicarle la carga registró el mejor comportamiento cuando tuvo pendientes entre 11 y $22^{\circ}$, pero a medida que iba aumentando la pendiente del talud, pasó a ser el suelo que peor recibía la carga del eucalipto. Este suelo presentó los picos más altos y bajos cuando actuó el nivel freático.

La arena mantuvo un beneficio de la carga hasta los $19^{\circ}$ de inclinación del talud, pero después no registró diferencias significativas de disminución del factor de seguridad a medida que aumentó la pendiente.

En los resultados obtenidos de la acción de la carga del eucalipto para taludes donde no se tuvo en cuenta el efecto del agua ni la cohesión añadida (véase figura 14), los factores de seguridad fueron disminuyendo sin importar el tipo de suelo a medida que aumentaba el ángulo de inclinación del talud; se destaca el menor efecto en la arena, contrario a la arcilla limosa, suelo que peor recibió la carga del eucalipto.

Al comparar con [10], hubo aportes positivos en ángulos mayores a $11^{\circ}$ de inclinación, algo que no ocurrió en este análisis (figura 14), aun compartiendo el hecho de que ninguno se hizo bajo los efectos del nivel freático.

Ningún suelo sin condiciones de presión de poros debido a la presencia de agua se comportó de manera benéfica en el talud al presentar decrecimientos en el factor de seguridad, sin embargo, es probable que el punto de quiebre pueda estar en un ángulo menor a $11^{\circ}$ de inclinación del talud.

En los resultados del artículo [10], los tres tipos de suelo analizados - los cuales son los mismos tomados en este estudio y las geometrías son las mismas, pero con una carga de $0,5 \mathrm{kPa}$ - mostraron un incremento en el factor de seguridad cuando se comparó con el suelo sin carga, por ejemplo, en la arena limosa hubo números positivos de incremento del F. S. hasta llegar a un intervalo entre los 22 y $27^{\circ}$ de inclinación del talud; en el caso de la arena, el estudio mostró números favorables al talud hasta un rango de inclinación entre los 19 y $22^{\circ}$, mientras que la arcilla fue la que más alcance en cuanto a pendiente del talud mostró: un rango entre los 27 y $34^{\circ}$, por el cual pasa de aportar a la estabilidad a ser un efecto nocivo para el mismo.

En este estudio se analizó con una carga de 2,5 kPa, $2 \mathrm{kPa}$ más que en [10] la cual se le aplicó al talud, dando resultados negativos de incremento al factor de seguridad en todos los casos (tabla 11). 


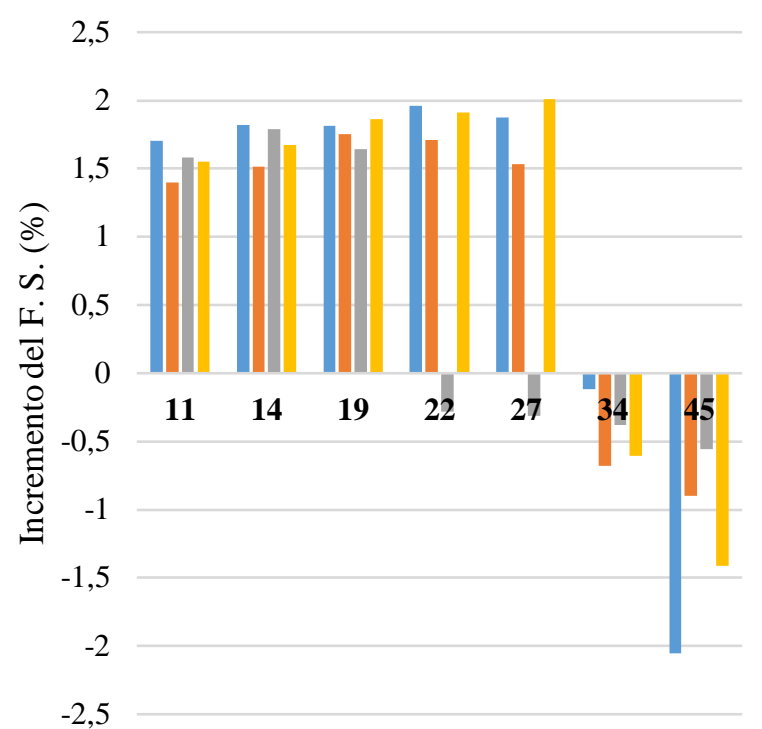

Ángulo del talud

$\square$ Arcilla Limosa $\square$ Arena Limosa $\square$ Arena $\square$ Arcilla

Figura 13. Efecto de la carga debida al árbol eucalipto dependiendo el tipo de suelo conforme el ángulo del talud cambia. Se tuvo en cuenta el nivel freático.

Tabla 11. Comparación con el artículo [10] sobre el rango entre pendientes de talud donde el factor de seguridad es 1 (ángulo neutro)

\begin{tabular}{|c|c|c|}
\hline \multicolumn{2}{|c|}{ Rango de pendientes donde F. S. $=\mathbf{1}^{\circ}$} \\
\hline Suelo & $\begin{array}{c}\text { Kokutse (2016) } \\
\text { Carga 0,5 kPa }\end{array}$ & $\begin{array}{c}\text { Artículo } \\
\text { Actual } \\
\text { Carga 2,5 kPa }\end{array}$ \\
\hline Arcilla & $19-22$ & Menos de 11 \\
\hline Arena & $27-34$ & Menos de 11 \\
\hline Arena limosa & $22-27$ & Menos de 11 \\
\hline
\end{tabular}

\subsection{Aporte al factor de seguridad por parte de las plantas}

Sin presencia de nivel freático (figura 15), el vetiver fue disminuyendo gradualmente su aporte al factor de seguridad a medida que incrementaba el ángulo de inclinación del talud, sin embargo, el aporte fue mucho mayor si se compara con los resultados que se obtuvieron del análisis por medio del equilibrio límite en presencia de nivel freático (figura 16), el cual a pesar de que la planta vetiver aportara crecimientos del factor de seguridad relativamente bajos, a medida que aumentaba el ángulo de inclinación, aumentaba su aporte al factor de seguridad.

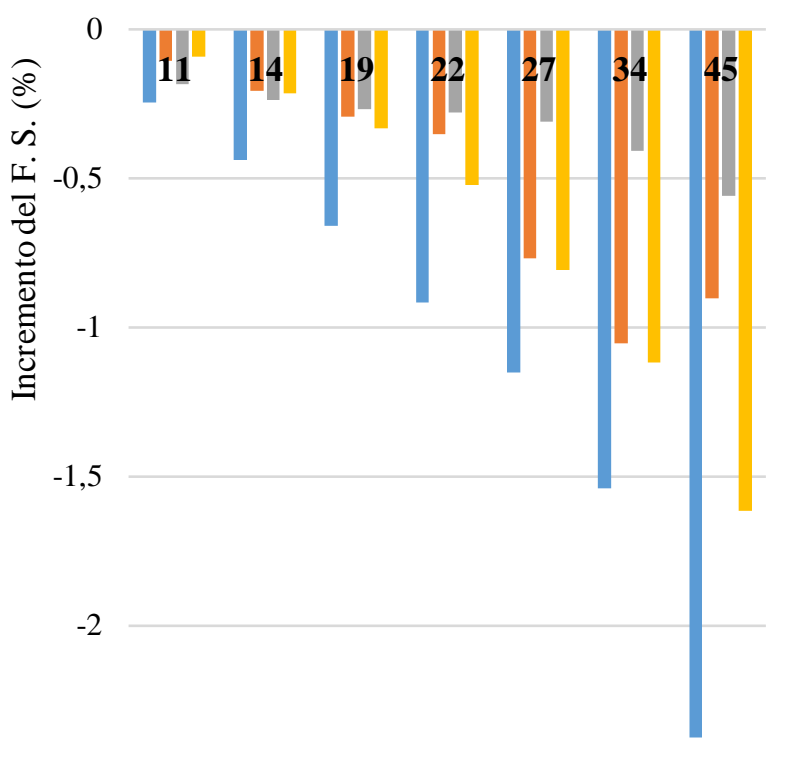

$-2,5$

Ángulo de inclinación del talud

n Arcilla Limosa $\square$ Arena Limosa $\square$ Arena $\backsim$ Arcilla

Figura 14. Influencia de la carga ocasionada por el eucalipto en el talud en F. S., sin tener en cuenta el efecto del agua, según cada tipo de suelo y con variación del ángulo de inclinación del talud.

Usando el eucalipto como estabilizador del talud, el factor de seguridad, independientemente de si había o no presencia de presión de poros, incrementó de manera gradual a medida que el ángulo de inclinación aumentaba, (véanse figuras 17 y 18), teniendo en cuenta que el aporte sin presencia de nivel freático fue mayor a cuando se tuvo en cuenta.

La tabla 12 representa el ángulo en el cual el factor de seguridad es de 1 para cada tipo de planta, teniendo en cuenta el nivel freático y basándose en un promedio entre todos los tipos de suelo y sus geometrías. 

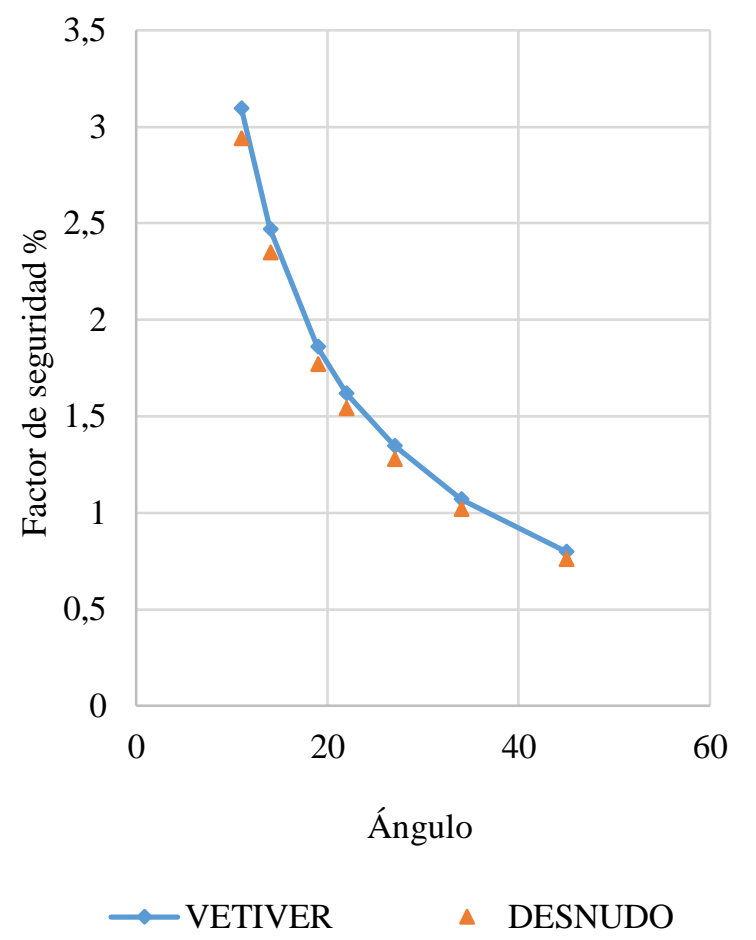

Figura 15. Decrecimiento del factor de seguridad a medida que incrementa la pendiente sin presencia de agua utilizando la planta vetiver.

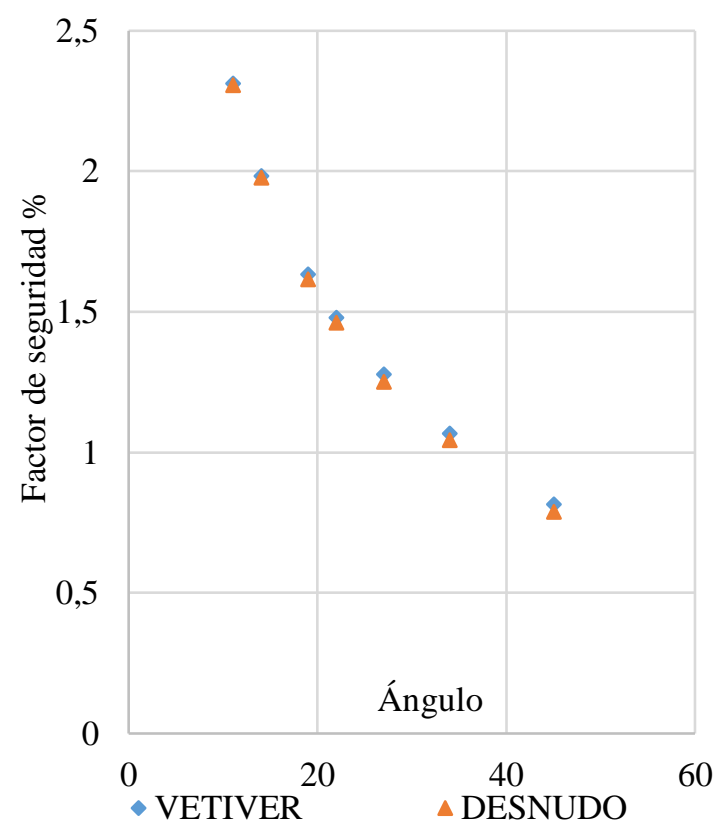

Figura 16. Decrecimiento del factor de seguridad a medida que incrementa la pendiente en presencia de agua utilizando la planta vetiver.

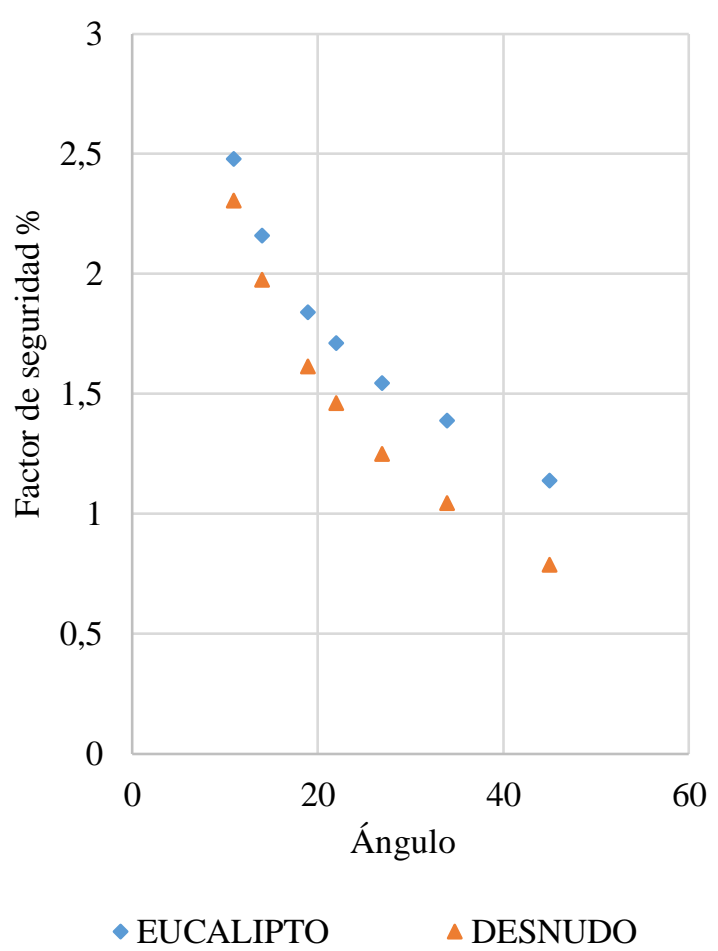

Figura 17. Decrecimiento del factor de seguridad a medida que incrementa la pendiente con presencia de agua utilizando el eucalipto.

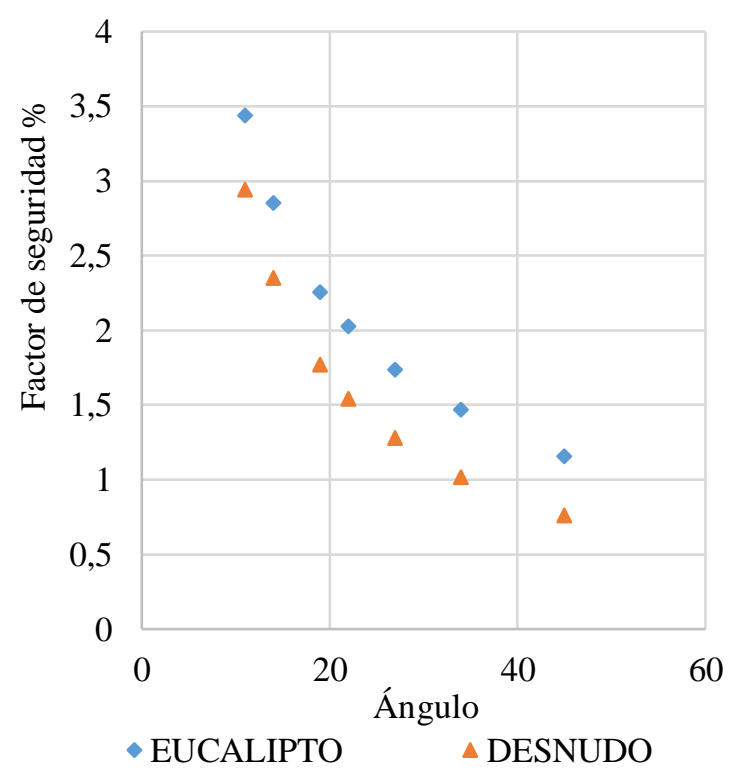

Figura 18. Decrecimiento del factor de seguridad en $\%$ a medida que incrementa la pendiente sin presencia de agua utilizando el eucalipto. 
Dicho ángulo incrementó un 5,33 \% con respecto a la superficie desnuda cuando se le aplicó el refuerzo generado por el vetiver en presencia de nivel freático; en cambio, sin presencia de nivel freático, este aumento fue del 13,161\%. Al usar el eucalipto, el ángulo límite donde el factor de seguridad es igual a 1 presentó un aumento del 45,2 \% comparándolo con el análisis a suelo desnudo en presencia de nivel freático; ahora, sin nivel freático, este aumento registró $59,96 \%$.

Tabla 12. Ángulos donde el factor de seguridad está a punto de fallar

\begin{tabular}{|c|c|c|}
\hline \multirow{2}{*}{ Planta } & \multicolumn{2}{|c|}{ Ángulo cuando el F. S.=1 } \\
\cline { 2 - 3 } & Con N. F. & Sin N. F. \\
\hline Vetiver & 36.262 & 39.699 \\
\hline Eucalipto & 49.988 & 55.066 \\
\hline Desnudo & 34.427 & 35.082 \\
\hline
\end{tabular}

\section{Discusión}

\subsection{Efecto del agua y de las plantas en el talud}

Las plantas ayudan a la estabilidad del talud independientemente de si existe un nivel freático, pero proporcionan un mayor crecimiento relativo del factor de seguridad cuando el suelo está seco; se aclara que no se tiene en cuenta el efecto de la evapotranspiración.

El vetiver joven aporta a la estabilidad del talud, pero este aporte es mínimo si se compara con el efecto del eucalipto. Debido a que el vetiver analizado se encuentra en una etapa donde se denomina joven, la cohesión que genera con las raíces al suelo puede cambiar con el paso del tiempo, por lo tanto, se recomienda en futuros estudios tener en cuenta un vetiver más consolidado. Sin embargo, es probable que el uso extendido del vetiver no se deba a su aporte a la estabilidad de deslizamientos rotacionales profundos, si no a movimientos traslacionales superficiales y a fenómenos de erosión superficial [26].

La tabla muestra que a pesar de que el vetiver genere un refuerzo mucho menor al eucalipto, puede llegar a incrementar el ángulo en el que falla a más de $3^{\circ}$ de pendiente sin presencia de nivel freático. Sin embargo, cuando existe agua en el talud, el cambio llega a ser casi insignificante. El eucalipto en cuanto a crecimiento de factor de seguridad tiene un comportamiento similar al vetiver, es decir, en suelo seco el aumento del factor de seguridad con respecto al suelo desnudo es s uperior al incremento del factor de seguridad del talud cuando el suelo está húmedo, no obstante, su aporte es mayor al del vetiver. Es importante señalar que la altura del árbol eucalipto no fue tenida en cuenta en este estudio.

\subsection{Peso del eucalipto}

En el análisis no se tuvo en cuenta los cambios físicos ni químicos de la planta con el tiempo, lo cual generaría variaciones en el factor de seguridad, por lo que se recomienda posteriormente realizar un análisis para los diferentes periodos de madurez de las plantas.

El efecto de la evapotranspiración potencial en el talud afecta el nivel de agua, el cual puede ocasionar cambios en los resultados del factor de seguridad. Al no tener en cuenta la transpiración potencial propia del eucalipto, el peso del eucalipto es un factor de riesgo para taludes con presencia de nivel freático debido a su negativa interacción con el suelo al no tener en cuenta la cohesión generada por las raíces del suelo (véase figura 14).

En comparación con el artículo de [10] cuyo análisis se basó en el modelo de elementos finitos por medio del programa Plaxis 2D, cuando se le aplicó la carga de 0,6 $\mathrm{kPa}$ por $\mathrm{m}^{2}$, en cada suelo hubo un cambio del factor de seguridad, el cual en un inicio colabora con la estabilidad hasta cierto punto donde empezaba a ser desfavorable para el talud; en cambio, con el peso promedio del eucalipto de 2,5 $\mathrm{kPa}$, el cual se analizó con el modelo de equilibrio límite mediante el programa Slide, no hubo tal cambio sin tener en cuenta el nivel freático: para cada suelo se comportó de forma negativa afectándolo. Esta comparación genera incertidumbre, por lo que se recomienda para futuras investigaciones un análisis de sensibilidad del factor de seguridad con respecto a la carga del talud.

\subsection{Efecto del agua y de las plantas en los diferentes tipos de suelos}

Los suelos en este estudio son afectados únicamente por agua subterránea, sin tener en cuenta el efecto del frente húmedo generado por lluvias que puede ser desencadenante de deslizamientos superficiales, por lo cual se recalca que este análisis está enfocado en deslizamiento rotacionales relativamente profundos. Se debe tener en cuenta que no todas las plantas serán buenas para todos los suelos, eso depende de muchos parámetros o factores no tenidos en cuenta en este estudio. En este caso solo se estudia el Chrysopogon zizanioides y el Eucalyptus por lo que se recomienda tener en cuenta plantas adicionales y ver su aporte a los 
diferentes suelos estudiados, por lo que es recomendable igualmente hacer análisis adicionales que contengan más cantidad de suelos a los analizados anteriormente además de incluir datos adicionales como el peso específico húmedo del suelo, lo cual no exime de realizar un análisis específico para cada talud real que se quiera tratar para comprobar cuál planta es más óptima para determinado tipo de suelo y mejorar el factor de seguridad de manera efectiva.

\section{Conclusiones}

El peso del eucalipto en caso de suelo húmedo hizo que el talud fallara entre los ángulos de 27 y $34^{\circ}$ para todos los suelos, excepto la arena, la cual registró la falla entre los 19 y $22^{\circ}$ de inclinación del talud. El incremento del factor de seguridad aumenta a medida que el ángulo de inclinación es mayor, hasta llegar al rango de falla registrado para cada suelo, lo cual evidencia una caída abrupta del incremento del factor de seguridad que pasa a ser negativo (véase figura 13).

La carga que proporciona el eucalipto al talud, en el cual se evidencia la presencia de agua, es peligrosa ya que hace disminuir considerablemente el factor de seguridad, sin embargo, el aporte de las raíces al suelo en términos de cohesión representa un incremento mayor que el efecto nocivo de la carga generada haciendo posible su uso como agente estabilizador de taludes.

Tanto en el vetiver como en el eucalipto se registró un incremento del factor de seguridad con respecto al suelo desnudo; en ausencia de nivel freático, se registró un incremento del factor de seguridad levemente mayor a cuando el nivel freático estuvo presente, sin embargo, para ambas plantas el porcentaje de incremento del factor de seguridad fue cada vez mayor a medida que el ángulo de inclinación aumentaba cuando en el talud había nivel freático, algo que no sucedió cuando el talud no presentaba nivel freático.

El eucalipto a diferencia del vetiver tiende a proporcionar más resistencia al suelo debido a que sus raíces son más profundas y proporcionan más cohesión que la brindada por el vetiver.

El eucalipto fue la planta que se vio más afectada por la presencia de agua y sin esta; a pesar de este comportamiento, fue la que dio al talud un mayor factor de seguridad comparado en estas condiciones al vetiver y a un suelo desnudo.
El eucalipto mostró un comportamiento de crecimiento gradual, que se atribuye a su gran capacidad como agente de refuerzo natural generando incrementos del factor de seguridad relativamente altos con diferencias que varían entre 1,7 y 11,7 puntos porcentuales.

La arena, debido a su cohesión casi nula, es más propensa al efecto de esfuerzo cortante del peso del eucalipto; este suelo empezó a tener disminuciones de factor de seguridad en un ángulo menor a los demás suelos (véanse figuras 13 y 14). El esfuerzo cortante en el talud debido a la carga vertical ofrecida por el eucalipto es mayor a medida que el ángulo aumenta, en suelos como la arcilla donde predomina la cohesión, sin presencia de agua, el talud empezó a mostrar disminuciones del factor de seguridad entre los ángulos 27 y 34, lo cual significa que un suelo cohesivo obtiene efectos positivos del peso de la planta a inclinaciones mayores a las que puede soportar un suelo no cohesivo como la arena. Cuando existe presencia de agua en el talud. El efecto del peso del eucalipto se ve mitigado por su gran aporte a la cohesión del suelo, pero si no existiera tal aporte en cohesión, la planta no serviría como estabilizador del terreno, debido a que su peso no genera más de 3 puntos porcentuales de efecto positivo o negativo al talud (véanse figuras 5 y 6), mientras que en el análisis en el cual se tuvo en cuenta la cohesión, los incrementos a la estabilidad del talud fueron entre 9 y $23 \%$ (véanse figuras 2 y 3).

Ambas plantas tienen presencia en Colombia, lo cual hace posible estabilizar taludes de forma ecológica tanto con el vetiver como con el eucalipto, las cuales demuestran su efectividad estabilizadora en los taludes con las características analizadas.

\section{Referencias}

[1] J. S. Xu, X. L. Yang, "Three-dimensional stability analysis of slope in unsaturated soils considering strength nonlinearity under water drawdown", Eng. Geol., vol. 237, pp. 102-115, 2018, doi: 10.1016/j.enggeo.2018.02.010.

[2] I. Khubulava, G. Chakhaia, "The forecast of stability of the landslide slope existing in the River Gldaniskhevi Valley," Ann. Agrar. Sci., vol. 16, no. 3, pp. 321-323, 2018, doi: 10.1016/j.aasci.2018.05.008.

[3] J. Suárez Díaz, "Prevención, Estabilización y Diseño", en Deslizamientos y estabilidad de taludes en zonas tropicales, 1998, pp. 385-428. 
[4] C. Sanhueza, G. Villavicencio, "Influencia de la cohesión aparente generada por raíces sobre la estabilidad de un talud natural en las dunas de Reñaca", Rev. la Constr., vol. 11, no. 1, pp. 16-31, 2012, doi: 10.4067/S0718-915X2012000100003.

[5] L. P. H. Van Beek, J. Wint, L. H. Cammeraat, J. P. Edwards, "Observation and simulation of root reinforcement on abandoned mediterranean slopes", en Eco y bioingeniería del suelo: el uso de la vegetación para mejorar la estabilidad de las pendientes. Desarrollos en Ciencias Vegetales y del Suelo, vol. 103, A. Stokes, I. Spanos, J. Norris, E. Cammeraat (eds) Springer, Dordrecht, 2007, doi: 10.1007/978-1-4020-5593-5_10.

[6] A. Paz Cardona, "Hace 9 meses estudios advertían de tragedia en Mocoa", Medio Ambiente, 2017 [En línea]. Disponible en: https://sostenibilidad.semana.com/medioambiente/articulo/mocoa-corpoamazonia-advirtio-de-latragedia-por-deforestacion/37471.

[7] H. Zhu, L. M. Zhang, T. Xiao, X. Y. Li, "Enhancement of slope stability by vegetation considering uncertainties in root distribution", Comput. Geotech., vol. 85, pp. 84-89, 2017, doi: 10.1016/j.compgeo.2016.12.027.

[8] K. H. Eab, S. Likitlersuang, A. Takahashi, "Laboratory and modelling investigation of rootreinforced system for slope stabilisation", Soils Found., vol. 55, no. 5, pp. 1270-1281, 2015, doi: 10.1016/j.sandf.2015.09.025.

[9] Y. Li et al., "Influence of the spatial layout of plant roots on slope stability", Ecol. Eng., vol. 91, pp. 477-486, 2016, doi: 10.1016/j.ecoleng.2016.02.026.

[10] N. K. Kokutse, "Slope stability and vegetation: Conceptual and numerical investigation of mechanical effects", Ecological Engineering, vol. 86, pp. 146-153, 2016, doi: 10.1016/j.ecoleng.2015.11.005.

[11] L. J. Escobar Toro, Y. Valencia González, "Análisis de estabilidad y probabilidad de falla de dos taludes de suelo tropical en la autopista Medellín-Bogotá en el tramo de vía entre marinilla y santuario", Boletín ciencias de la tierra, 2012 [En línea]. Disponible en: https://revistas.unal.edu.co/index.php/rbct/article/view/3 $1253 / 43364$.

[12] N. S. Nilaweera, P. Nutalaya, "Role of tree roots in slope stabilisation", Bull. Eng. Geol. Environ., vol. 57, no. 4, pp. 337-342, 1999, doi: 10.1007/s100640050056.
[13] G. Flórez, "Efectividad de la bioingeniería para el tratamiento de la erosión y los movimientos en masa en laderas", tesis de maestría, Universidad de Manizales, 2014.

[14] J. E. Benavides, "Arboles y arbustos forrajeros: una alternativa agroforestal para la ganadería", en FAO animal production and health paper, 1999, pp. 367-390.

[15] Food and Agriculture Organization of the United Nations (FAO), El eucalipto en la repoblación forestal. 1981.

[16] D. H. Ashton, "The root and shoot development of Eucalyptus regnans F. Muell”, Aust. J. Bot., vol. 23, no. 6, pp. 867-887, 1975, doi: 10.1071/BT9750867.

[17] U. Salazar Gómez, "El vetiver agarra el suelo Flojo", El Colombiano, 2011, [En línea]. Disponible en: http://www.elcolombiano.com/historico/el_vetiver_agar ra_el_suelo_flojo-DGec_121637.

[18] R. Grimshaw, "Vetiver, un excelente pasto para la conservación del agua", LEISA, 1999, [En línea]. Disponible en: http://www.leisaal.org/web/index.php/volumen-14-numero-1/2499vetiver-un-excelente-pasto-para-la-conservacion-delagua.

[19] L. A. Bárcenas Montenegro, L. R. Díaz Martínez, G. L. Flores Baquedano, "Evaluación de la actividad antioxidante de 12 especies vegetales", tesis de grado, Universidad Nacional Autónoma de Nicaragua, 2012.

[20] P. Hurtado, A. L. U. Z. Villa, F. R. Durán, "Estudio de mercado de aceite esencial de naranja en Colombia en el período 2009-2014", Revista Colombiana de Ciencias Hortícolas, vol. 10, no. 2, pp. 301-310, 2014, doi: 10.17584/rcch.2016v10i2.4653.

[21] S. B. Mickovski, A. Stokes, R. van Beek, M. Ghestem, T. Fourcaud, "Simulation of direct shear tests on rooted and non-rooted soil using finite element analysis", Ecol. Eng., vol. 37, no. 10, pp. 1523-1532, 2011, doi: 10.1016/j.ecoleng.2011.06.001.

[22] T. C. T. Hubble, D. W. Airey, H. K. Sealey, E. V. De Carli, S. L. Clarke, “A little cohesion goes a long way: Estimating appropriate values of additional root cohesion for evaluating slope stability in the Eastern Australian highlands", Ecol. Eng., vol. 61, pp. 621-632, 2013, doi: 10.1016/j.ecoleng.2013.07.069. 
[23] S. B. Mickovski, L. P. H. van Beek, "Root morphology and effects on soil reinforcement and slope stability of young vetiver (Vetiveria zizanioides) plants grown in semi-arid climate", Plant Soil, vol. 324, no. 1, pp. 43-56, 2009, doi: 10.1007/s11104-009-0130-y.

[24] T. C. T. Hubble, B. B. Docker, I. D. Rutherfurd, "The role of riparian trees in maintaining riverbank stability: A review of Australian experience and practice", Ecol. Eng., vol. 36, no. 3, pp. 292-304, 2010, doi: 10.1016/j.ecoleng.2009.04.006.

[25] T. C. T. Hubble, I. D. Rutherfurd, "Evaluating the relative contributions of vegetation and flooding in controlling channel widening: The case of the Nepean River, Southeastern Australia", Aust. J. Earth Sci., vol. 57, no. 5, pp. 525-541, 2010, doi: 10.1080/08120099.2010.492910.

[26] T. T. Van, P. Truong, "R \& D Results on Unique Contributes of Vetiver Applicable for Its Use in Disaster Mitigation Purposes in Vietnam", en First Indian National Vetiver Workshop, Cochin, India, 2008, pp. 103-115. 